\title{
Comparison of Delay-Interferometer and Time- Lens-Based All-Optical OFDM Demultiplexers
}

\author{
Lillieholm, Mads; Mulvad, Hans Christian Hansen; Galili, Michael; Oxenløwe, Leif Katsuo
}

Published in:

IEEE Photonics Technology Letters

Link to article, DOI:

10.1109/LPT.2015.2412882

Publication date:

2015

Link back to DTU Orbit

Citation $(A P A)$ :

Lillieholm, M., Mulvad, H. C. H., Galili, M., \& Oxenløwe, L. K. (2015). Comparison of Delay-Interferometer and Time- Lens-Based All-Optical OFDM Demultiplexers. IEEE Photonics Technology Letters, 27(11), 1153-1156. https://doi.org/10.1109/LPT.2015.2412882

\section{General rights}

Copyright and moral rights for the publications made accessible in the public portal are retained by the authors and/or other copyright owners and it is a condition of accessing publications that users recognise and abide by the legal requirements associated with these rights.

- Users may download and print one copy of any publication from the public portal for the purpose of private study or research.

- You may not further distribute the material or use it for any profit-making activity or commercial gain

- You may freely distribute the URL identifying the publication in the public portal 


\title{
Comparison of Delay-Interferometer and Time- Lens-Based All-Optical OFDM Demultiplexers
}

\author{
Mads Lillieholm, Hans Christian H. Mulvad, Michael Galili and Leif K. Oxenløwe
}

\begin{abstract}
In this paper we present the first detailed numerical comparison of two promising all-optical schemes to demultiplex orthogonal frequency-division multiplexing (OFDM) signals. The investigated schemes are the optical discrete Fourier transformation (O-DFT) and the optical spectral magnification (SM) based on time lenses. In the former scheme, cascaded delayinterferometers (DIs) are used to perform the O-DFT, with subsequent active optical gating to remove the intercarrier interference (ICI). Here a reduced-complexity partial O-DFT, realized by replacing a number of DIs with optical bandpass filters, is investigated. In the latter scheme the OFDM spectrum is magnified, allowing for simple optical bandpass filtering of the individual subcarriers with reduced ICI. Ideally only a single unit consisting of two time lenses is needed, reducing the complexity and potentially the energy consumption compared to the type of O-DFT scheme relying on many active gates. The bit-error-rate is estimated down to $\sim 10^{-6}$ by Monte Carlo biterror counting for a 32-subcarrier OFDM input signal, showing that a performance close to the ideal O-DFT is achievable for both the reduced-complexity $\mathrm{O}-\mathrm{DFT}$ and the SM scheme.
\end{abstract}

Index Terms-All-optical OFDM, optical discrete Fourier transformation, optical Fourier transformation, time lens

\section{INTRODUCTION}

$I^{2}$ NCREASING internet capacity demands require energyefficient solutions, to obtain more favorable scaling of the energy consumption with the provided capacity. It is estimated that $2 \%$ of the world-wide $\mathrm{CO} 2$ emissions can be attributed to the power usage of internet systems equipment, which is equivalent to the contribution from the aviation industry [1]. Moreover, the capacity of wavelength-division multiplexing superchannels will be limited by currently available optical fiber bandwidth. Hence, to reduce the power consumption and need for new fiber installations, it is essential to develop lowenergy methods in support of optical signals with high spectral efficiency (SE), including energy-efficient signal processing.

In recent years, optical orthogonal frequency-division multiplexing (OFDM) has received interest for allowing high SE by intercarrier interference (ICI)-free demultiplexing of orthogonal subcarriers with considerable spectral overlap. For

Mads Lillieholm*, Michael Galili, and Leif K. Oxenløwe are with DTU Fotonik, Dept. of Photonics Engineering, Technical University of Denmark, Ørsteds Plads 343, 2800 Kgs. Lyngby, DK (e-mail: madsl@fotonik.dtu.dk*). H.C. Mulvad was with DTU Fotonik, Technical University of Denmark. Copyright (c) 2015 IEEE. Personal use of this material is permitted. However, permission to use this material for any other purposes must be obtained from the IEEE by sending a request to pubs-permissions@ieee.org. example, all-optical OFDM (AO-OFDM) demultiplexing techniques using optical signal processing (OSP) have been demonstrated by realizing the optical discrete Fourier transform (O-DFT), e.g. using arrayed waveguide grating routers (AWGRs) [2] or cascaded delay-interferometers (DIs) [3], in conjunction with ultrafast optical sampling. Coherent opto-electronic techniques with fast electronic sampling have also been proposed, e.g. employing a wavelength selective switch to perform the O-DFT [4], or using digital signal processing (DSP) to demultiplex electronically. To decrease the complexity of DI-based O-DFT schemes, a simplified approach, where several DIs are replaced with optical bandpass filters (OBPFs) to form a partial O-DFT, has been proposed [3]. Cascaded DIs require elaborate control schemes for phase-stabilization [5], although using an AWGR instead may require only precise temperature control, whereas OBPFs are simple, passive devices. In a novel scheme, a spectral telescope based on two active optical time lenses is used to magnify an OFDM spectrum, allowing low-complexity subcarrier demultiplexing by direct bandpass filtering with reduced ICI, compared to no magnification. The scheme has been proposed and demonstrated for a factor 4 magnification of a 28 subcarrier OFDM signal with $12.5 \mathrm{GHz}$ spacing [6], and using time lenses based on four-wave mixing.

It is the aim of this study to estimate the bit-error rate (BER) performances for the above-mentioned AO-OFDM receivers based on idealized implementations of the spectral telescope and of the partial O-DFT. For both receiver types, the reduced complexity comes at the cost of a small degree of ICI, depending on the magnification factor or complexityreduction level. Results are compared to the ideal O-DFT which in principle provides optimal, ICI-free recovery of OFDM subcarriers. For an input OFDM signal with 32 $10-\mathrm{GBd}$ subcarriers, it is found that the time time-lens-based receiver with magnification factor $M=8$ and the partial O-DFT-based receiver using only 15 instead of 31 DIs, corresponding to a complexity-reduction of $\theta=1$ in the nomenclature of this paper, can obtain similar performances, close to that of the ideal O-DFT. Assuming optical sampling using e.g. electro-absorption modulators (EAMs), the O-DFT requires 32 optical sampling gates in addition to 15 DIs, hence making the time-lens-based demultiplexer with only two active FWM devices less complex by comparison. However, the results herein assume ideal FWM time-lenses with rectangular pump pulses synchronized with the OFDM signal, and assumptions on the relative complexities are based on 
optical sampling as a requirement, to perform the signal processing in the optical domain.

\section{PRINCIPLE OF OPERATION}

The ideal OFDM signal consists of $N$ subcarriers with sincprofiles in the spectral domain, and frequency spacing $\Delta f=1 / T$, where $T$ is the symbol period. The OFDM signal can be generated optically or electro-optically using the inverse DFT (IDFT), which transforms parallel input data streams into OFDM symbols, in conjunction with parallel-to-serial conversion [7]. ICI-free recovery of the subcarriers is in principle possible using the DFT; in practice a cyclic prefix is inserted to help compensate for dispersion and other linear impairments [7]. In section B, an OFDM demultiplexing method using a telescopic time-lens-based approach is shown.

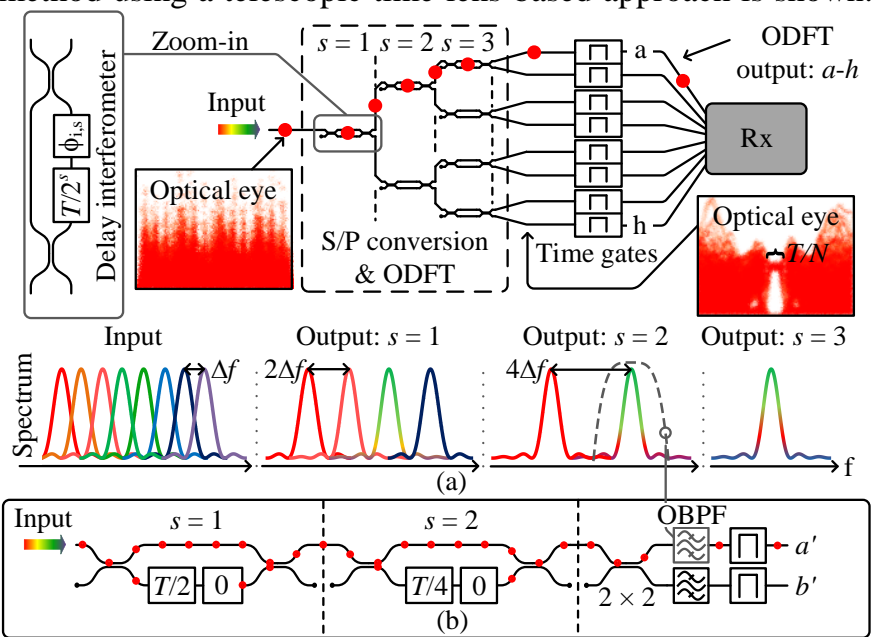

Fig. 1. Schematic illustration of the principle of an all-optical OFDM receiver based on an 8-point ODFT (a). Also shown is an example of a partial-ODFT, replacing one stage of DIs with OBPFs instead. (b)

\section{A. ODFT-Based AO-OFDM Receiver Principle}

Fig. 1(a) shows the principle for an $N=8$ subcarrier optical OFDM signal, with O-DFT and time gating, followed by demodulation and detection at the receiver. The demultiplexed subcarriers are labeled $a-h$, with the upper path for $a$ traced by dots. The 8-point O-DFT has 3 stages of DIs, numerated by $s$, and the spectral outputs for each of the stages are sketched. The $i$ th DI of a stage delays the lower-arm signal by $T / 2^{s}$ and incurs a relative phase shift $\phi_{\mathrm{i}, \mathrm{s}}$. Significant ICI remains after the O-DFT, but the interference is displaced temporally, resulting in an interference-free window which may be gated.

Fig. 1(b) shows the upper path of an 8-point partial O-DFT, retrieving subcarriers $a^{\prime}$ and $b^{\prime}$, corresponding to $a$ and $b$ for the ideal ODFT. Here, the third DI-stage has been replaced by $2 \times 2$ couplers and an OBPF per subcarrier. The dashed transfer function for the OBPF of $a^{\prime}$ is exemplified above, over the sketched spectral output for $s=2$ in Fig. 1(a). Designating the number of removed stages as $\theta$, the DI complexity reduction becomes $N-N / 2^{\theta}$ out of the $N-1$ used for an ideal $N$-point O-DFT. ICI is introduced for the partial O-DFT, as well as intersymbol interference (ISI) due to the limited OBPF bandwidth. Lowering the DFT-order may reduce the complexity for an O-DFT based on an AWGR as well, assuming that fewer arms require precise temperature control.

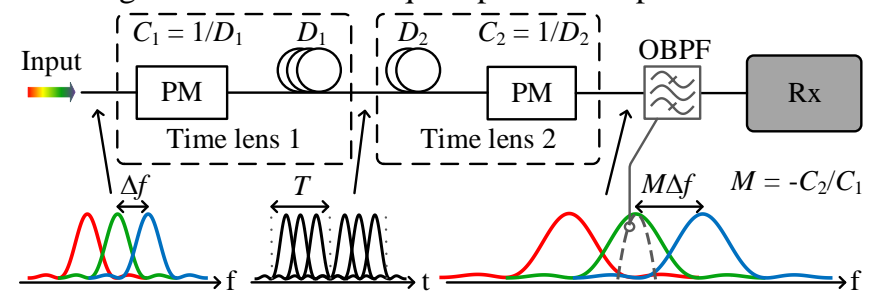

Fig. 2. The principle of all-optical OFDM demultiplexing based on spectral magnification: Chirp rate $C$, dispersion $D=1 / C$ and magnification $M$.

\section{B. Time-Lens-Based AO-OFDM Receiver Principle}

Using optical time lenses to magnify the OFDM spectrum, a subcarrier can be demultiplexed using only an OBPF. A time lens is a quadratic phase modulator (PM), which in conjunction with dispersion can manipulate the temporal and spectral profiles of an optical field to obtain an optical Fourier transformation (OFT) [6][8]. The principle of the time-lensbased OFDM receiver is illustrated in Fig. 2, showing different subcarriers mapped to different points in time by frequency-to-time (f-t) conversion, with subsequent time-tofrequency $(\mathrm{t}-\mathrm{f})$ conversion into the $\times \mathrm{M}$ magnified OFDM spectrum. A subcarrier is retrieved with an OBPF as indicated by the dashed transfer function. Note that applying a narrow bandpass filter (bandwidth $<1 / \mathrm{T}$ ) to the unmagnified input to avoid significant ICI will result in excessive ISI. Applying a chirp rate $C$ with subsequent accumulated chromatic dispersion $D=\beta_{2} L$, transfers the spectral profile into the time domain, if the OFT condition $C=1 / D$ is met; $\beta_{2}$ is the group velocity dispersion parameter, and $L$ the length of the dispersive medium. Conversely, dispersion followed by quadratic phase modulation, transfers the temporal profile to the spectral domain. By performing a f-t conversion using $C_{1}=1 / D_{1}$ with subsequent $\mathrm{t}-\mathrm{f}$ conversion using $C_{2}=1 / D_{2}$, the input spectral width $\Delta \omega_{\text {in }}$ relates to the output spectral width by $\Delta \omega_{\text {out }}=-C_{2} / C_{1} \Delta \omega_{\text {in }}$, yielding the magnification $M=-C_{2} / C_{1}$.

\section{Simulation SeTUP}

\section{A. OFDM Signal Generation Setup}

Fig. 3(a) shows the setup for the generation of a 32subcarrier OFDM signal. The subcarriers are generated individually with $10-\mathrm{GHz}$ frequency spacing and zero relative phase, with 100-ps width rectangular symbols independently modulated using DPSK or DQPSK. The subcarriers are multiplexed to obtain $10-\mathrm{GHz}$ subcarrier spacing, centered at $1543 \mathrm{~nm}$ with aggregate symbol rate of $320 \mathrm{GBd}$. The data of the evaluated subcarriers have PRBS length of $2^{10}-1$, whereas other subcarriers are modulated using random, uniformly distributed sequences. The simulated field has 1023 symbols with 256 samples per symbol at $10 \mathrm{GBd}$, for time and frequency resolutions of $0.4 \mathrm{ps}$ and $10 \mathrm{MHz}$ respectively.

\section{B. Ideal and Partial O-DFT-Based OFDM Receiver Setup}

The setup for the O-DFT-based receiver is shown in Fig. 3(b). Gaussian noise is added directly to the transmitted OFDM signal, which is demultiplexed by the O-DFT using 5- 

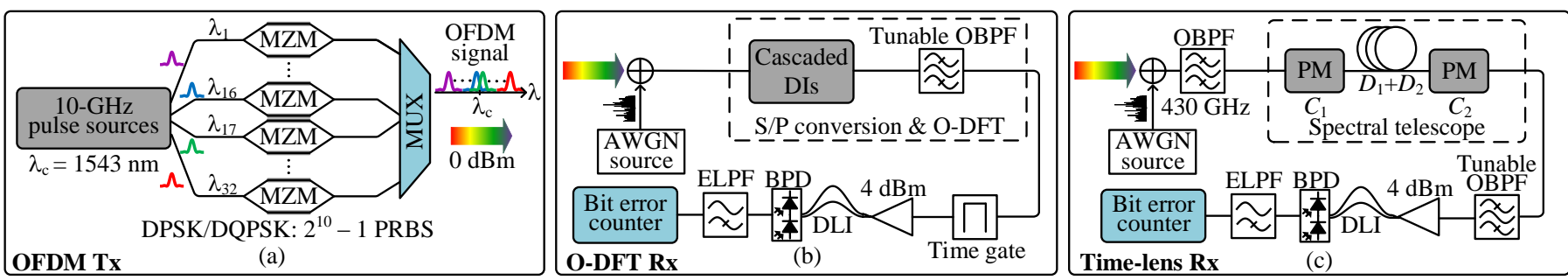

Fig. 3. Simulation setup diagrams: Optical OFDM transmitter (a), time-lens-based OFDM receiver (b), and O-DFT-based receiver (c). Note that DLI implies either DPSK or DQPSK demodulation. Abbreviations: AWGN: Additive white Gaussian noise. BPD: Balanced photo-diode. DLI: Delay-line interferometer. ELPF: Electrical lowpass filter. MZM: Mach-Zehnder modulator. PRBS: Pseudorandom binary sequence. S/P: Serial-to-parallel.

stage cascaded DIs, with variable complexity-reduction $0 \leq \theta \leq 5$ ( $\theta=0$ being ideal). A 10th order super Gaussian (SG) OBPF with variable 3-dB bandwidth optimized for each $\theta$, removes out-of-band noise which is not filtered by the cascaded DIs. The filter order is unrealistic, and is meant as an idealization. Lower order filters are found to achieve better performance, with similar gains for the partial O-DFT and the time-lens receiver described in section C. Placing the filter after the O-DFT was verified to perform similarly to a placement before. Subsequently, an ideal time gate removes the time-displaced ICI. The time gated subcarrier is demodulated, and detected using ideal balanced detection. The electrical signal is lowpass-filtered using a 7.5-GHz, 4th order Bessel filter. Finally, the BER is estimated by Monte Carlo simulations with bit-error counting. Sequences of 1023 symbols are repeated for different noise samples, and the accumulated error count is stored for all 256 sampling times with 40 decision thresholds each. When the minimum number of observed errors is $\geq 100$, a preliminary BER is estimated at the sampling point with the lowest error count. The final BER is obtained by averaging 5 preliminary BER estimates. Counting up to 100 errors yields reduced accuracy at low OSNR, but tests have reproduced the quantum limit with $95 \%$ confidence, for an ideal ASE-limited receiver with a matched optical filter and no electrical lowpass filter.

\section{Time-Lens-Based OFDM Receiver Setup}

Fig. 3(c) shows the simulation setup for the time-lens-based receiver. Gaussian noise is added to the input OFDM signal, and bandpass filtered by a rectangular, 430-GHz OBPF at the input to the spectral telescope. Here, ideal quadratic PM with fixed chirp rate $C_{1}=0.034 \mathrm{ps}^{-2}$ is applied, followed by accumulated chromatic dispersion $D_{1}+D_{2}$. $D_{1}$ is fixed by the OFT condition $D_{l}=1 / C_{1}$. $D_{2}$ depends on the chirp rate of the second PM, set to obtain a magnification factor of $M$. The telescope output is the input OFDM spectrum magnified $M$ times, where a subcarrier is filtered directly using a 10th order SG OBPF with optimized 3-dB bandwidth depending on $M$. Noise loading, demodulation, detection, and error counting is performed identically to the O-DFT-based receiver.

\section{Simulation Results}

Fig. 4 shows QPSK constellation diagrams for the complex field of central subcarrier 16 before demodulation, for both receiver types. Note that no noise has been added, and visible degradations are mainly due to ICI. Magnification factors 2, 4, and 8 are shown (left) alongside $\theta=0,1,2$ (right). The middle constellation shows direct bandpass filtering of the OFDM subcarrier, equivalent to $M=1$ or $\theta=5$ without a time gate. The ideal O-DFT enables optimal subcarrier demultiplexing, in principle without any ICI, given an ideal input OFDM signal. However, the bandpass filter removing out-of-band noise smears the constellation slightly. For $\theta=1$ and $\theta=2$ the partial O-DFT adds ICI, resulting in greater smearing of the constellation diagrams. Minimal ISI is added due to the large OBPF bandwidth for these $\theta$. For the time-lens-based receiver the constellations are clearly improved for larger $M$, which can be attributed to the reduced ICI.

Fig. 5 shows the estimated BER for both receiver types as a function of the OSNR per subcarrier. Results for $M=1$ and $\theta=4$ are inside the inset. The results shown are for subcarrier 16, which serves as a worst-case scenario with respect to ICI, for DPSK or DQPSK demodulation. Included for reference are the performances for single-subcarrier signals using matched OBPFs (no ICI with optimal filtering) [6], marking lower BER bounds. Shown below on Fig. 5, is the optimized 3-dB OBPF width as a function of $M$ for the time-lens receiver, as well as the optimized $3-\mathrm{dB} \mathrm{OBPF}$ and time gating window widths as a function of $\theta$ for the O-DFT receiver. The large difference in optimum bandwidth between DPSK and DQPSK for $M=8$ is due to a decreasing BER variation with bandwidth for increasing $M$, so that similar performance is obtained over a wide range of bandwidths. At the bottom of Fig. 5, the OSNRpenalties compared to the ideal O-DFT with respect to the conventional FEC-threshold, BER $=3.8 \times 10^{-3}$ are shown, in addition to penalties at $\mathrm{BER}=10^{-5}$, representing more relaxed FEC requirements. At $\mathrm{BER}=10^{-5}$ penalties of $0.2 \mathrm{~dB}$ and $0.7 \mathrm{~dB}$ are observed for $\theta=1$, for DPSK and DQPSK respectively, relative to the ideal $\mathrm{O}-\mathrm{DFT}$ reference $(\theta=0)$. For the time-lens receiver, the penalties become $0.2 \mathrm{~dB}$ for DPSK and $0.5 \mathrm{~dB}$ for DQPSK for $M=8$. Hence the ICI becomes very small for $M \geq 8$. Note that whereas clear optimum time gate widths for the partial O-DFT exist, the penalty for increasing the width by as much as $50 \%$ is on the order of $0.2 \mathrm{~dB}$ with respect to the FEC-threshold for DPSK with $\theta=1-3$, and a $3-4 \mathrm{~dB}$ penalty for removing the time gate entirely. For DQPSK the FEC-threshold can be reached for $\theta=1-3$, although not without a time gate, in which case the penalties range from $0.3 \mathrm{~dB}(\theta=1)$ to $3.7 \mathrm{~dB}(\theta=3)$ for a $50 \%$ time gate width deviation. Hence, the time gate requirements may be relaxed for the partial O-ODFT, which is promising with respect to further complexity reduction potential.

The components necessary to achieve $M=8$ are the same as for lower magnification factors, although the system 

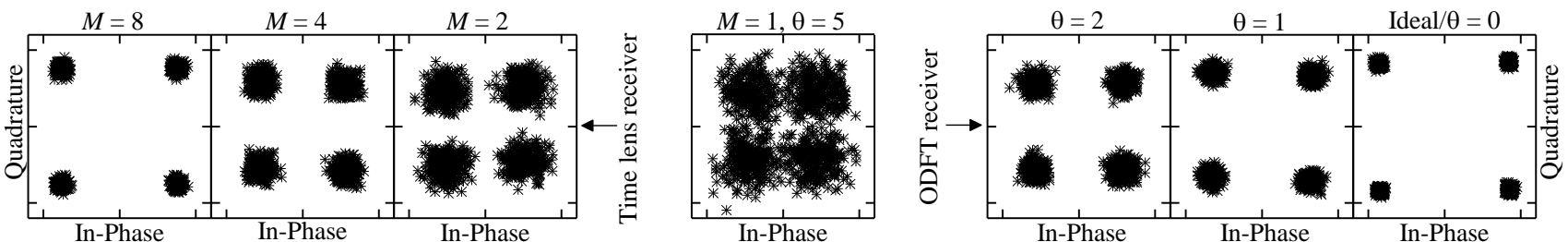

Fig. 4. ASE-noise-free QPSK constellation diagrams for the time-lens-based receiver for $M=2$ to $M=8$ (left), and for the ODFT-based receiver in the ideal case as well as for $\theta=1$ and $\theta=2$ (right). The middle diagram shows $M=1$ and $\theta=5$, corresponding to direct bandpass filtering. All diagrams show subcarrier 16 .
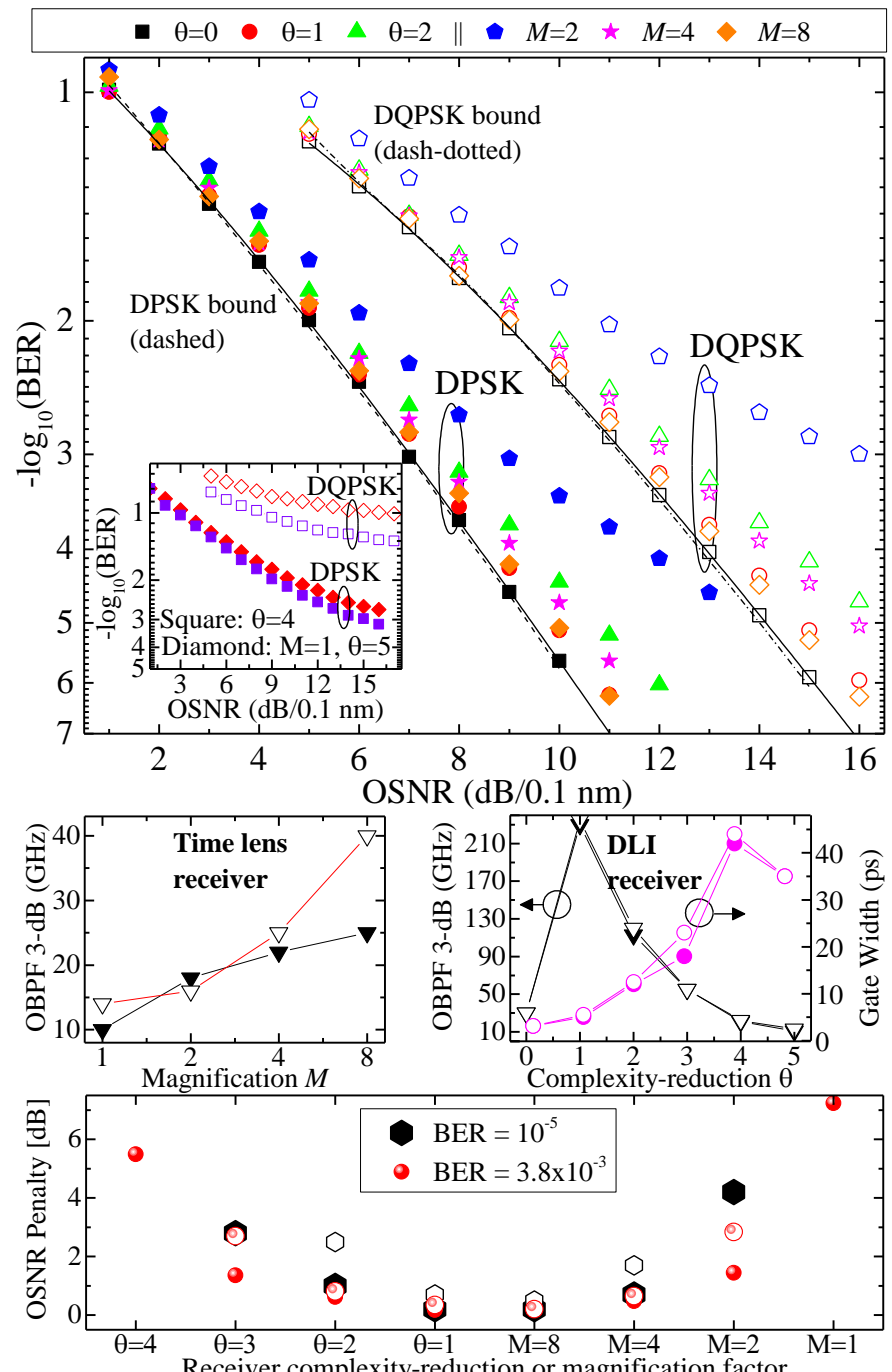

Fig. 5. Simulated $-\log (B E R)$ vs. OSNR for selected O-DFT-based and timelens-based receiver parameters (top) and OSNR-penalties (bottom). Also shown is the OBPF 3-dB bandwidth as a function of $M$ (left), and the OBPF 3 $\mathrm{dB}$ bandwidth (triangles) and gating window width (circles) as a function of $\theta$, on the left and right axes respectively (right). Results are for subcarrier 16 with DPSK (solid symbols) and DQPSK (open symbols) demodulation.

requirements increase due to increased FWM conversion bandwidth and pump bandwidth demands. Compared to coherent receivers with DSP for electronic synchronization, dispersion compensation and demultiplexing, there are many practical challenges for the time-lens and partial O-DFT receivers relying entirely on OSP. In general, DSP offers solutions with much lower complexity than OSP; however, it is the potential to go beyond the bandwidth limitations and power consumption of high-speed electronics driving the OSP interest. Although the electronic bandwidth requirements can be relaxed by combining OSP and DSP, e.g. using the O-DFT in conjunction with electronic gating [4], it is important to investigate all-optical solutions in pursuit of practical methods for the elimination of electronic bottlenecks.

\section{CONCLUSION}

The BER vs. OSNR performance of two all-optical OFDM demultiplexing schemes has been investigated down to a BER 10 $0^{-6}$ by Monte Carlo simulations, using a 32-subcarrier OFDM signal encoded with DPSK or DQPSK as input. Compared to the ideal O-DFT demultiplexer requiring $31 \mathrm{DIs}$, a partial O-DFT-based demultiplexer with only 15 DIs $(\theta=1)$ and an optical bandpass filter per subcarrier, shows near-ideal performance. A similar performance is achieved for a timelens-based demultiplexer with a spectral magnification factor of 8 , and subsequent optical bandpass filtering of subcarriers. For a fully OSP-based receiver, the partial O-DFT requires ultrafast optical sampling with an inverse scaling of the gating window width with the number of subcarriers, e.g. using a high-bandwidth electro-absorption modulator per subcarrier. On the other hand, the time-lens-based demultiplexer requires only two active FWM-devices, and therefore benefits from a lower complexity and a potential energy consumption scaling which does not necessarily scale with the number of subcarriers. Hence, these simulations indicate that the partial O-DFT and time-lens-based schemes can achieve highperformance, all-optical demultiplexing of OFDM signals.

\section{REFERENCES}

[1] C. A. Chan et al., "Methodologies for Assessing the Use-Phase Power Consumption and Greenhouse Gas Emissions of Telecommunications Network Services", Environ. Sci. Technol., vol. 47, no. 1, pp. 485-492, Jan. 2013

[2] A. J. Lowery, "Design of arrayed-waveguide grating routers for use as optical OFDM demultiplexers," Opt. Express, vol. 18, no. 13, pp. 14129-14143, Jun. 2010.

[3] D. Hillerkuss et al., "Simple all-optical FFT scheme enabling Tbit/s realtime signal processing," Opt. Express, vol. 18, no. 9, pp. 9324-9340, Apr. 2010.

[4] J. Schröder et al., "Reconfigurable all-optical Discrete Fourier Transform in a wavelength selective switch for optical OFDM demultiplexing”, OFC, Los Angeles, USA, OTh1G.6, Mar. 6-10, 2012.

[5] K. Takiguchi et al., "PLC-based eight-channel OFDM demultiplexer and its demonstration with $160 \mathrm{Gbit} / \mathrm{s}$ signal reception", OFC, San Diego, USA, OThB4, Mar. 21-25, 2010.

[6] P. Guan et al., "All-optical OFDM System using a Wavelength Selective Switch based Transmitter and a Spectral Magnification based Receiver," ECOC, Cannes, France, Tu.3.6.2, Sep. 21-25, 2014.

[7] J. Armstrong, "OFDM for Optical Communications", Opt. Express, vol. 27, no. 3, pp. 189-204, Feb. 2009.

[8] H.C. H. Mulvad et al., "Ultra-high-speed optical serial-to-parallel data conversion by time-domain optical Fourier transformation in a silicon nanowire," Opt. Express, vol. 19, no. 26, pp. B825-B835, Dec. 2011 